\title{
Aldosterone as a Mediator of Progressive Renal Dysfunction: Evolving Perspectives
}

\author{
Murray EPSTEIN
}

\begin{abstract}
End-stage renal disease (ESRD) comprises an enormous public health burden, with an incidence and prevalence that are increasingly on the rise. This escalating prevalence suggests that newer therapeutic interventions and strategies are needed to complement current therapeutic approaches. Although much evidence demonstrates conclusively that angiotensin II mediates progressive renal disease, recent evidence also implicates aldosterone as an important pathogenetic factor in progressive renal disease. Recently, several lines of experimental evidence demonstrate that selective blockade of aldosterone, independent of renin-angiotensin blockade, reduces proteinuria and nephrosclerosis in the spontaneously hypertensive stroke-prone rat (SHRSP) model and reduces proteinuria and glomerulosclerosis in the subtotally nephrectomized rat model (ie, remnant kidney). Whereas pharmacologic blockade with angiotensin II receptor blockers and angiotensin-converting enzyme (ACE) inhibitors reduces proteinuria and nephrosclerosis/glomerulosclerosis, selective reinfusion of aldosterone restores these abnormalities despite continued renin-angiotensin blockade. Aldosterone may promote fibrosis by several mechanisms, including plasminogen activator inhibitor-1 (PAI-1) expression and consequent alterations of vascular fibrinolysis, by stimulation of transforming growth factor-beta1 (TGF- $\beta 1$ ), and by stimulation of reactive oxygen species (ROS). Based on this formulation, randomized clinical studies will be initiated to delineate the potential renal-protective effects of aldosterone receptor blockade.
\end{abstract}

(Internal Medicine 40: 573-583, 2001)

Key words: aldosterone receptor antagonist, renin-angiotensin system, end-stage renal disease (ESRD), diabetic nephropathy, plasminogen activator inhibitor-1 (PAI-1)
Introduction

End-stage renal disease (ESRD) comprises a tremendous public health burden, with an incidence and prevalence that are rapidly increasing. As an example, during 1998 the incidence of new cases of ESRD in the United States was 85,520, with a total prevalence in the United States of nearly 324,000 (1). Only 3 years prior to this, in 1995 , the US prevalence was 257,266 (2). In fact, the prevalence of ESRD has increased every year since 1988 , nearly doubling over the past decade (1). Worldwide, the experience has been similar in other countries including Japan (3). The two most important causes of ESRD are diabetic nephropathy and hypertension, and the incidence attributable to each continues to rise substantially each year. This escalating prevalence of ESRD translates into a major societal burden as exemplified by dramatically increasing expenditures for medical care and a substantive impairment in the quality of life of the ESRD population. Consequently, major clinical and investigative initiatives have focused on developing newer therapeutic interventions and strategies to complement current approaches.

\section{Pathogenesis}

To adequately address the treatment and prevention of ESRD, we must first determine, and then appropriately manage, the causative mechanisms involved. The mediators of progressive renal disease have not been fully established, but include a wide array of vasoactive hormones, growth factors, and cytokines including the renin-angiotensin-aldosterone system (RAAS), endothelin, transforming growth factor- $\beta 1$ (TGF$\beta 1$ ), and others that have been considered in detail in several recent reviews (4-6).

The RAAS is an essential causative link between hypertension and ESRD. Clinical trials (7-8) have repeatedly demonstrated that interruption of this system dramatically reduces renal injury in patients with hypertension and with diabetes. Although angiotensin II is often the primary mediator of the RAAS that is associated with renal disease progression, a number of studies (9-14) have raised the possibility that aldosterone per se is etiologic in mediating progressive renal disease.

From Department of Medicine, University of Miami School of Medicine, Miami, Florida Reprint requests should be addressed to Dr. Murray Epstein, MD, FACP, Nephrology Section, VA Medical Center, 1201 NW $16^{\text {th }}$ Street, Miami, FL 33125 
As detailed in a recent review (15), these observations include studies in experimental models such as the spontaneously hypertensive stroke-prone rat (SHRSP) and remnant kidney model. Consequently, clinical studies are now being performed to verify the value of aldosterone receptor blockade in improving hypertension-related outcomes, with specific attention to improvements in end-organ protection including the kidney (16).

This review will focus on emerging evidence that aldosterone, independent of renin-angiotensin, may participate in mediating progressive renal disease. Additionally, the emerging therapeutic implications of aldosterone receptor blockade will be discussed.

\section{Traditional concepts: renin-angiotensin-aldosterone system (RAAS)}

A number of experimental models, including the subtotal renal ablation model (remnant kidney model), have demonstrated the effects of hypertension in progressive renal disease. Systemic hypertension promotes increased glomerular hydrostatic pressure and increased nephron plasma flow, both of which promote glomerular hyperfiltration and, ultimately, proteinuria and progressive renal failure. Several investigators utilizing diverse experimental models have shown that not all antihypertensive agents that adequately control systemic pressure are able to reduce glomerular capillary pressure $\left(\mathrm{P}_{\mathrm{GC}}\right)$ or attenuate renal injury $(17,18)$. Because it is the increased pressure in the glomerular capillary that is the major mediator of renal disease progression, it is at this point that investigators have focused their investigative efforts to interrupt the cycle of progressive renal injury. It was proposed that such pharmacologic intervention would prevent, and even reverse, disease progression.

Studies in animal models have shown that angiotensin-converting enzyme (ACE) inhibitors block the generation of angiotensin II, with a consequent decrease in angiotensin II in the renal vasculature, attenuating efferent arteriolar constriction and, thus, glomerular capillary pressure (17-19). Based on this theoretic construct, a number of clinical trials $(7,8)$ have established that interruption of the RAAS cascade with ACE inhibitors is beneficial in limiting renal disease progression. These trials have validated the concept that counteracting angiotensin II by pharmacologic blockade results in attenuation of progressive renal disease. As early as 1993, in the Diabetes Collaborative Study (8), Lewis et al examined whether captopril has renal-protective effects in diabetic nephropathy, independent of its capacity to lower blood pressure. In this trial, patients with insulin-dependent diabetes mellitus, a urinary protein excretion $\geq 500 \mathrm{mg} / \mathrm{day}$, and a serum creatinine $\leq 2.5$ $\mathrm{mg} / \mathrm{dl}$ were treated with captopril or placebo. The primary endpoint was a doubling of the baseline serum creatinine concentration. Compared with placebo, patients treated with captopril demonstrated a $50 \%$ reduction in the risk of death, dialysis, and renal transplantation that was independent of the difference in blood pressure between groups. This study was the first major investigation to underscore the preferential renal- protective effect of ACE inhibitors that is independent of bloodpressure lowering.

Subsequently, the Ramipril Efficacy in Nephropathy (REIN) core study (7) examined the use of an ACE inhibitor, ramipril, on the progression of nondiabetic nephropathy. In this study, ramipril was compared with conventional antihypertensive treatment (dosed to achieve DBP $<90 \mathrm{mmHg}$ ) in reducing the rate of decline in the glomerular filtration rate (GFR), reducing proteinuria, and preventing ESRD. The results demonstrated that in patients with proteinuria $\geq 3 \mathrm{~g} / \mathrm{day}$, the monthly decline in GFR with ramipril was significantly lower than with placebo $(p=0.03)$, and the risk of renal disease progression was significantly reduced even after adjustment for changes in blood pressure ( $\mathrm{p}=0.04)$. In the subsequent REIN follow-up trial (20), ramipril prevented the decline in GFR over time and eliminated the need for dialysis after a treatment period of $\geq 3$ years in patients with chronic nephropathy. Finally, ACE inhibition has been shown to confer a renoprotective effect in patients with nondiabetic renal disease (21). These few studies exemplify what many recent studies have collectively documented, i.e., the renoprotective effects mediated by blockade of the renin-angiotensin system.

\section{Emerging Concepts: The Role of Aldosterone in Mediating Progressive Renal Disease}

Although much evidence has accumulated to implicate angiotensin II in mediating renal disease, recent evidence also suggests that aldosterone is an important factor in causing progressive renal disease through both hemodynamic effects and direct cellular actions (Table 1) $(10,22,23)$. Obviously this concept constitutes a paradigm shift, differing from the longheld notion that aldosterone acts primarily as a sodium-retaining hormone (24-26). In the following sections, I will review recent and emerging evidence indicating that aldosterone may promote deleterious effects on both the cardiovascular system and the kidney.

\section{Traditional concept}

In its capacity as a mineralocorticoid hormone, aldosterone has receptor-ligand endocrine properties on epithelial cell sodium and potassium exchange in classic target tissues, such as kidneys, colon, and salivary and sweat glands (24-26). Its significance during dietary sodium deprivation or in response to salt and water loss is clear and can be life saving. Its contribution to the pathophysiologic origins of such salt avid states as congestive heart failure, cirrhosis, and nephrotic syndrome is well established. In recent years, aldosterone's endocrine properties have taken on a broader perspective involving nonclassic actions in nonepithelial cells found in nonclassic target tissues. These actions likewise contribute to the pathophysiology of cardiac fibrosis and cardiovascular dysfunction $(27,28)$.

Several lines of evidence point to a major role of aldosterone in promoting progressive renal dysfunction. Observational studies in patients with primary aldosteronism (PA) suggest a pathogenetic role for hyperaldosteronism. Originally it was 
Table 1. Relationship Between Augmented Aldosterone Levels and Progressive Renal Disease (10, 22, 23)

Remnant Kidney Model

- Untreated remnant rats had ten-fold higher elevations in aldosterone levels Greene et al (10) and a greater degree of proteinuria and glomerulosclerosis than shamoperated rats $(\mathrm{p}<0.05)$

- Proteinuria and glomerulosclerosis were reduced in remnant rats treated with losartan and enalapril versus untreated remnant rats $(\mathrm{p}<0.05)$

- Selective infusion of aldosterone to remnant rats treated with losartan and enalapril restored proteinuria and glomerulosclerosis to an extent similar to that seen in untreated remnant rats

Clinical Studies in Patients with Chronic Renal Insufficiency

- Eight normokalemic patients with chronic renal failure (mean $\mathrm{CL}_{\mathrm{CR}} 14.3$ $\mathrm{ml} / \mathrm{min}$ ) had elevated aldosterone levels

- While ingesting a normal sodium intake, 5 of 8 patients had elevated aldosterone levels

- While ingesting a low sodium intake, all 7 patients had elevated aldosterone levels

- Aldosterone levels were assessed in 28 patients with varying degrees of renal insufficiency who had normal serum potassium levels and plasma renin activity

- All 28 patients with renal insufficiency had increased aldosterone levels compared with 22 healthy volunteers

- Aldosterone levels were highest among patients with the greatest impairment in renal function $\left(\mathrm{CL}_{\mathrm{CR}} 3\right.$ to $10 \mathrm{ml} / \mathrm{min}$ )

$\mathrm{CL}_{\mathrm{CR}}$ : creatinine clearance. Adapted from Epstein (15).

postulated that hypertensive patients with low levels of plasma renin activity have fewer cardiovascular complications than those with normal or elevated levels of plasma renin activity. However, in several recent studies, cardiovascular complications were found in $14 \%$ to $35 \%$ of the patients with PA. Moreover, in patients with PA, the incidence and degree of proteinuria was reported to be greater than that in patients with essential hypertension (29-31).

In analogy with these observations in PA patients, observations in chronic renal insufficiency support this formulation. Clinical studies $(22,23)$ have also demonstrated a relationship between the augmented levels of aldosterone and renal deterioration. Berl et al (22) found that plasma aldosterone levels were elevated in 5 of 8 normokalemic patients with renal failure, and in 5 of 6 patients with a creatinine clearance of $<15$ $\mathrm{ml} / \mathrm{min}$. In a subsequent study by Hene et al (23), plasma aldosterone levels of 28 patients with creatinine clearances $<50 \%$ of normal were increased, despite normal serum potassium levels and normal plasma renin activity. It is likely that potassium and angiotensin II - both at increased levels in patients with renal failure - act synergistically to promote the aldosterone excess that accompanies renal insufficiency and progressive renal disease (5).

A number of experimental models are consistent with the concept that aldosterone may play a pathogenetic role in medi- ating renal injury. Hyperaldosteronism and adrenal hypertrophy are common findings in the remnant kidney model, with plasma levels of aldosterone increased approximately ten fold (10). In a study by Quan et al (13), hypertension, proteinuria, and structural renal injury were less prevalent in rats that underwent subtotal nephrectomy with adrenalectomy compared with rats that had partial nephrectomy but intact adrenal glands. This occurred despite large doses of replacement glucocorticoid (aldosterone was not replaced) in the adrenalectomized rats.

In the deoxycorticosterone acetate (DOCA)-salt hypertensive rat model, exogenous administration of mineralocorticoids induced lesions of malignant nephrosclerosis and stroke (9). The development of this type of lesion is likely due to the intrinsic action of mineralocorticoids, as these rats demonstrate low levels of plasma renin activity and responded poorly to ACE inhibitor therapy. Horiuchi et al (11) found an increased concentration of aldosterone receptors in the kidneys of a substrain of stroke-prone spontaneously hypertensive rats (MSHRSP), in which the development of malignant nephrosclerosis occurred without salt loading. Furthermore, Ullian et al (14) demonstrated that Wistar-Furth rats - which are unresponsive to the actions of aldosterone - are resistant to developing nephropathy in response to subtotal nephrectomy.

Although many studies have demonstrated a beneficial ef- 
fect of ACE inhibition in retarding progressive renal disease, it must be emphasized that this intervention does not differentiate between the relative contribution of renin-angiotensin versus aldosterone. To evaluate the possible contribution of aldosterone per se, Rocha et al conducted a series of experiments in SHRSP that succeeded in dissociating the relative contributions of aldosterone and the renin-angiotensin system $(12,32)$. Initially, they implanted time-release pellets of spironolactone, an aldosterone receptor antagonist, or placebo pellets in saline-drinking $(1 \% \mathrm{NaCl})$ SHRSP ingesting a Stroke-Prone Rodent diet (32). This model is known to induce severe hypertension and glomerular and vascular lesions characteristic of thrombotic microangiopathy as observed in malignant nephrosclerosis. Blood pressure and urinary protein excretion were assessed for a 3- to 4-week period. They demonstrated that mineralocorticoid receptor blockade with spironolactone markedly attenuated urinary protein excretion $(150 \mathrm{mg} /$ day for the placebo group vs $39 \mathrm{mg} /$ day for the spironolactone group, $\mathrm{p}<0.0001$ ). Proteinuria remained at baseline levels in the spironolactone group approximately 12 weeks later, although urinary protein excretion remained elevated in placebo-implanted animals (136 mg/day for the placebo group vs $39 \mathrm{mg} /$ day for the spironolactone group, $\mathrm{p}<0.0001$ ) (Fig. 1) (32). Histologic examinations revealed fewer nephrosclerotic and cerebrovascular lesions in the spironolactone group than in the placebo group ( $p<0.01$ and $p<0.001$, respectively). Notably, systolic blood pressure did not differ between the two groups at any time during the study (see Fig. 1).

In a subsequent study, Rocha et al (12) investigated whether an aldosterone infusion would reverse the renal-protective effects of captopril therapy in SHRSP. The study divided SHRSP into five groups: vehicle (control), captopril (50 mg/kg/day), aldosterone infusion $(40 \mu \mathrm{g} / \mathrm{kg} /$ day $)$, or captopril $(50 \mathrm{mg} / \mathrm{kg} /$ day) with aldosterone infusion (at 20 and $40 \mu \mathrm{g} / \mathrm{kg} / \mathrm{day}$ ). Data from this analysis are shown in Fig. 2 (12). Animals in the control and aldosterone infusion groups experienced marked proteinuria and comparable degrees of renal injury $(21 \%$ and $29 \%$, respectively). In contrast, captopril treatment reduced endogenous aldosterone levels, prevented the development of proteinuria, and prevented the development of glomerular and renal vascular lesions. However, subsequent aldosterone infusion reversed the ability of captopril to confer this protection. The aldosterone-infused, captopril-treated rats demonstrated proteinuria, renal vascular lesions, and glomerular lesions despite ACE inhibitor treatment. Importantly, systolic blood pressure in captopril-treated SHRSP receiving the aldosterone infusion was not significantly different than in SHRSP treated with captopril alone. Thus, the renal injury induced by aldosterone was independent of blood pressure increases, suggesting a more direct tissue effect of aldosterone.

This concept has been extended to other experimental models including the remnant kidney model. In the following experiments, as in the above studies, the role of aldosterone has been dissociated from that of angiotensin II in the progression of renal disease. Greene et al (10) evaluated four treatment groups (sham-operated rats, untreated remnant rats, remnant rats treated with losartan and enalapril, and remnant rats treated with losartan and enalapril followed by an infusion of aldosterone) to distinguish the relative importance of aldosterone in the progression of renal injury. They observed that remnant rats had a ten-fold elevation in aldosterone levels in comparison with sham-operated rats. In contrast, remnant rats under-

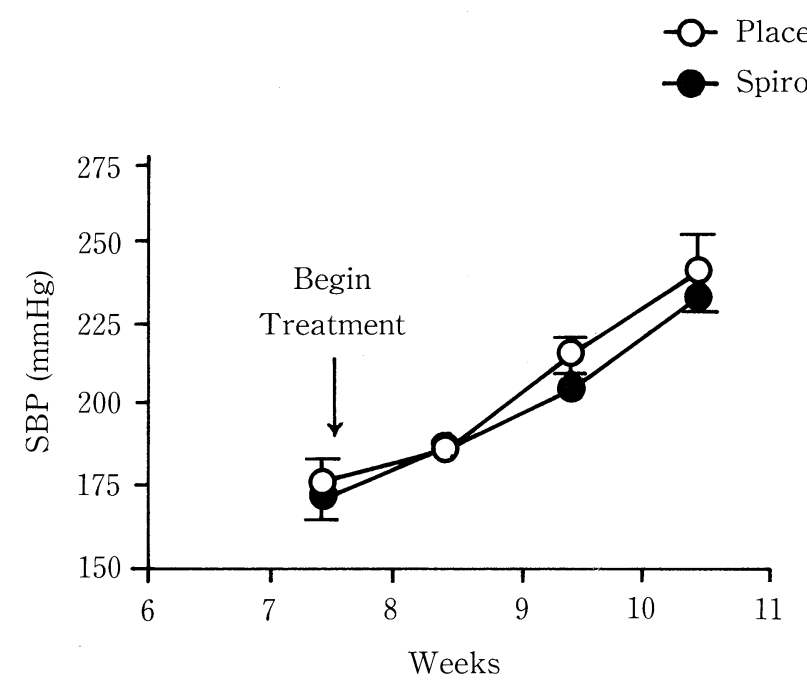

A

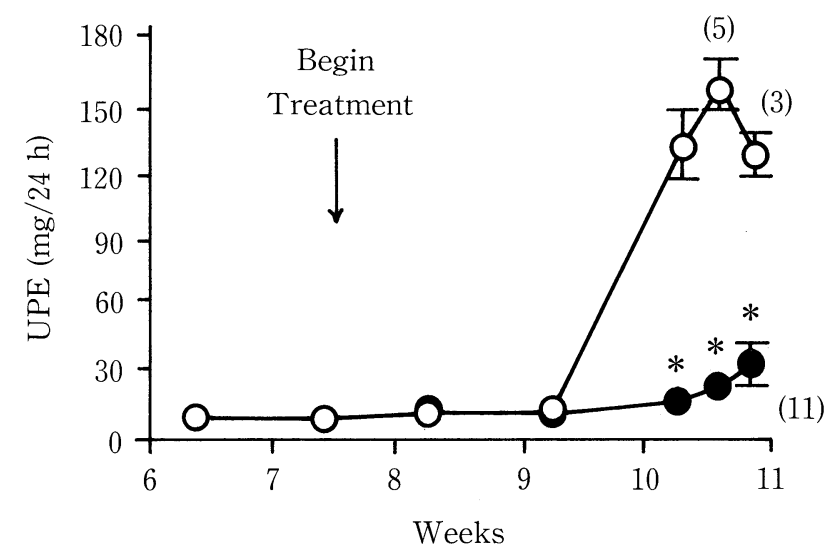

B

Figure 1. Effect of spironolactone on urinary protein excretion in stroke-prone spontaneously hypertensive rats (SHRSP). Line graphs showing (A) systolic arterial blood pressure (SBP) and (B) urinary protein excretion (UPE) in SHRSP into which timerelease pellets containing spironolactone or placebo were implanted. Values are mean \pm SEM. Numbers in parentheses indicate the number of animals. ${ }^{*} \mathbf{p}<0.001$ compared with placebo-implanted controls. [Adapted, with permission, from Rocha et al (32)]. 


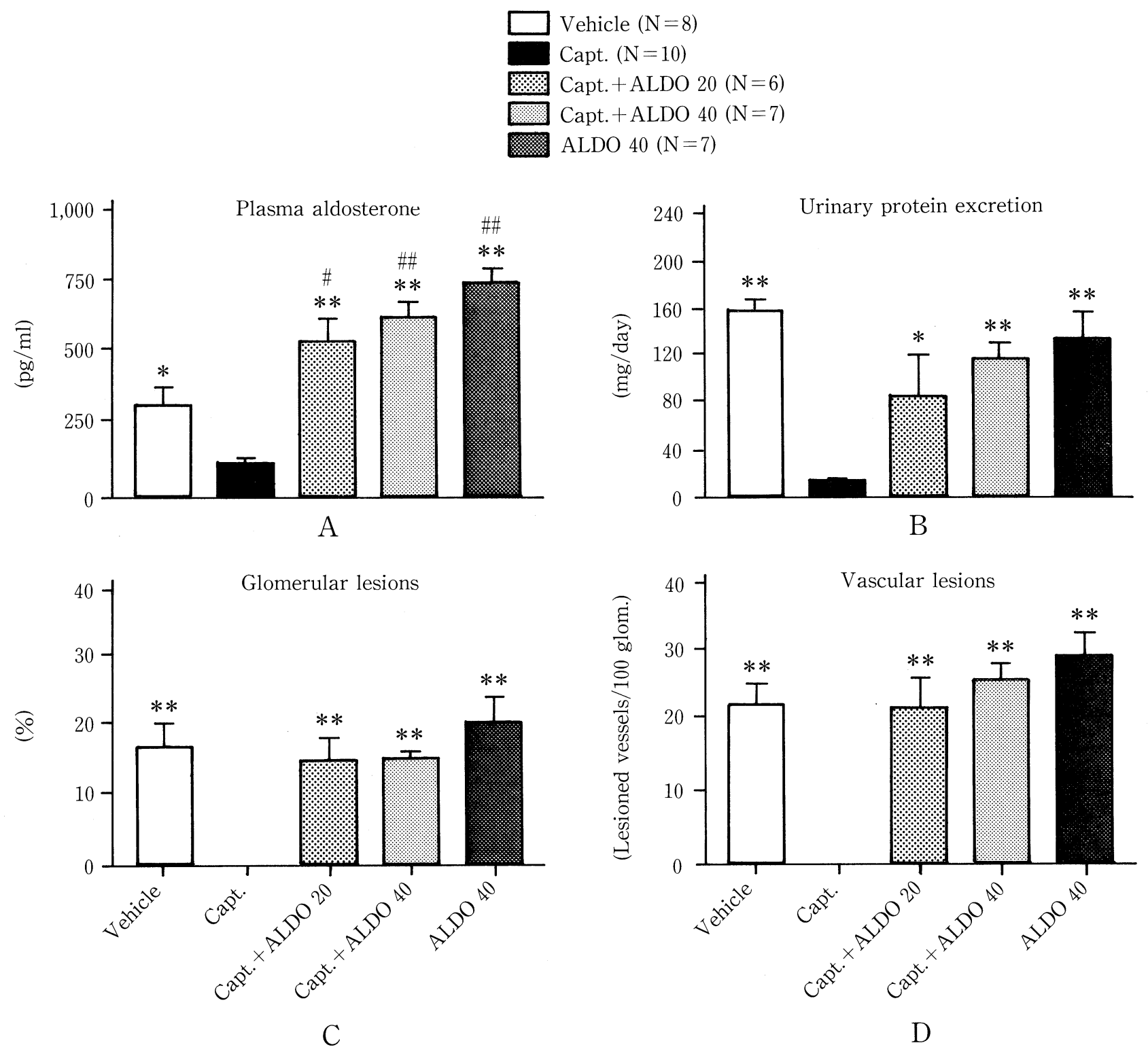

Figure 2. Effect of aldosterone blockade on urinary protein excretion and glomerulopathy in stroke-prone spontaneously hypertensive rats (SHRSP). Bar graphs showing plasma aldosterone (A), urinary protein excretion (B), glomerular lesions (C), and renal vascular lesions (D) in captopril-treated, saline-drinking SHRSP at the end of 2 weeks of treatment with aldosterone or vehicle. ${ }^{*} \mathbf{p}<0.01,{ }^{*} *_{\mathrm{p}}<0.001$ compared with captopril; ${ }^{\#} \mathbf{p}<0.01,{ }^{\#} \mathbf{p}<0.001$ compared with vehicle. Values are mean \pm SEM: capt, captopril, ALDO: aldosterone. [Adapted, with permission from, Rocha et al (12)].

going treatment with losartan and enalapril manifested suppressed aldosterone levels, with a decrease in proteinuria, hypertension, and glomerulosclerosis compared with the remnant rats not given these agents. In the final group of remnant rats receiving losartan and enalapril treatment followed by an infusion of aldosterone, the degree of proteinuria, hypertension, and glomerulosclerosis was similar to that of untreated remnant rats. These results further support an independent pathogenetic role for aldosterone as a mediator of progressive renal disease.
Of note, in a recent preliminary communication, Hostetter et al (33) reported that aldosterone antagonists did not provide nephroprotection in the remnant kidney model despite a significant decrease in left ventricular hypertrophy. The reasons for the discrepancy between the remnant kidney model (33) and the SHRSP model (32) are not readily apparent. As detailed in a recent review (15), a number of methodological differences may account for the discrepant results including differences in potassium intake and renin-angiotensin system activation. 


\section{Extrarenal Adverse Effects of Aldosterone: Implications for Progressive Renal Injury}

It should be emphasized that the data supporting a pathogenetic role for aldosterone are not limited to the kidney, but rather, a generalized phenomenon. For several years, mineralocorticoids, including aldosterone, have also been implicated in the injury to extrarenal components of the vascular system. A review of this evidence provides additional insight into the potential mechanisms involved in aldosterone-mediated renal injury.

Numerous studies over the past 10 years suggest that the nonepithelial actions of mineralocorticoids are responsible for their vascular and myocardial fibrotic and trophic effects (3436). Although the primary site of pharmacologic action of aldosterone is at the mineralocorticoid receptors in the epithelium of the distal nephron, colon, and rectum, receptors also have been located on nonepithelial sites in the blood vessels, brain, and heart (37-40). Furthermore, sites of aldosterone formation outside of the adrenal gland have been discovered, including human endothelial cells and vascular smooth muscle cells (VSMCs) (41), and within myocardial cells in animal studies (42).

Aldosterone has recently been hypothesized to exert a direct cellular effect to induce fibrosis and hypertrophy in VSMCs and myocardial cells - effects that may previously have been inaccurately attributed solely to the systemic hypertension caused by mineralocorticoids (Table 2$)(27,35,43-50)$. Several studies $(34,36)$ have linked mineralocorticoids with myocardial fibrosis via stimulation of collagen formation in myocardial cells.

Circulating aldosterone may mediate vascular fibrosis by the direct interaction of this steroid hormone with high affinity, low-capacity corticoid receptors that are located in the cytosol of vascular fibroblasts. When activated, the receptor loses its heat shock protein, and its monomeric form reaches the cell nucleus, where it binds to DNA with its binding region to initiate the expression of mRNA for type I collagen (or other proteins) synthesis (51).
Another mechanism whereby aldosterone may promote fibrosis in several target organs, including the kidney, centers on its effects on the plasminogen activator system (see Table 2). The effects of the RAAS on the plasminogen activator system serves as one of the major endogenous defense mechanisms against intravascular thrombosis and also plays an important role in vascular and tissue remodeling. Vascular fibrinolytic balance is, to a large part, determined by the competing effects of plasminogen activators and plasminogen activator inhibitor-1 (PAI-1), both of which are locally synthesized in the blood vessel wall, and in particular, the vascular endothelium.

Nakamura et al (52) have hypothesized that a major component of the vascular toxicity brought on by activation of the RAAS is derived from the deleterious effects of angiotensin on fibrinolytic balance. There is a growing body of evidence, including data from the molecular/cellular level, experimental studies in animal, and clinical studies, indicating that ACE is strategically located to regulate vascular fibrinolytic balance.

Recently, Brown and colleagues (47) have proposed that aldosterone contributes to the regulation of PAI-1 expression. Utilizing transfected rat aortic smooth muscle cells and cultured human umbilical vein endothelial cells, they demonstrated that aldosterone modulates angiotensin II-induced PAI-1 expression. Furthermore, they demonstrated that the effect of aldosterone on PAI-1 synthesis was attenuated by spironolactone. Finally, to determine whether aldosterone affected PAI-1 expression in vivo, they measured local venous PAI-1 antigen concentrations in 6 patients with primary hyperaldosteronism undergoing selective adrenal vein sampling. Concentrations of PAI-1 antigen, but not tissue plasminogen activator antigen, were significantly higher in adrenal venous blood than in peripheral venous blood. Taken together, these data support the hypothesis that aldosterone modulates the effect of angiotensin II on PAI-1 expression in vitro and in vivo in humans. Furthermore, recent data $(47,48)$ indicate that plasma PAI-1 levels are closely correlated with serum aldosterone in salt-depleted healthy subjects and that aldosterone enhances the induction of PAI-1 expression in smooth muscle cells in vitro. Recent in vivo data indicate that aldosterone activity, as assessed by serum aldosterone levels, correlates with PAI-1 antigen levels,

Table 2. Potential Mechanisms Whereby Aldosterone Mediates Fibrosis and Collagen Formation $(27,35,43-50)$

- Upregulation of angiotensin II receptors (35)

- Potentiation of the pressor responses of angiotensin II (35)

- Increases in sodium influx in vascular smooth muscle cells (44)

- Inhibition of norepinephrine uptake in vascular smooth muscle cells and myocardial cells $(43,45)$

- Participation in vascular smooth muscle cell hypertrophy (46)

- Modulation of the effect of angiotensin II on plasminogen activator inhibitor-1 (PAI-1) expression $(47,48)$

- Stimulation of transforming growth factor beta-1 (TGF- $\beta 1$ ) synthesis (50)

- Generation of reactive oxygen species (ROS) (49)

- Promotion of endothelial dysfunction (27)

Adapted from Epstein (15) 
supporting an interaction between aldosterone and the fibrinolytic system (53). As a result, it is probable that angiotensin and aldosterone likely act in concert to regulate vascular fibrinolysis and tissue remodeling. Consequently, studies should be initiated to investigate the effects of specific aldosterone antagonists on PAI-1 production, and the effects of such interventions on resultant renal dysfunction.

Ullian et al (35) demonstrated that aldosterone may promote VSMC hypertrophy by inducing upregulation of angiotensin II receptors, thus potentiating the pressor responses of angiotensin II - an effect that was largely inhibited by spironolactone.

Recently, Sun et al (50) have reported studies indicating that aldosterone may exert its deleterious effects on the kidney through stimulation of TGF- $\beta 1$ synthesis. They demonstrated that uninephrectomized rats receiving aldosterone $(0.75 \mu \mathrm{g} / \mathrm{h}$, subcutaneously) and $1 \% \mathrm{NaCl}$ in drinking water for 6 weeks developed diffuse fibrosis in both the renal cortex and medulla and abundant myofibroblasts were noted at these sites of fibrosis. These changes were associated with significantly increased binding density of ACE and angiotensin II receptors (60\% AT1 receptors, $40 \%$ AT2 receptors) at the sites of fibrosis and markedly increased expression of TGF- $\beta 1$ and type I collagen mRNAs at these same sites. In summary, in this rat model of renal repair, the enhanced expression of ACE, angiotensin II receptors, and TGF- $\beta 1$ is associated with renal fibrosis. Angiotensin II generated at the sites of repair appears to have autocrine/paracrine functions in the regulation of renal fibrous tissue formation alone or through its stimulation of TGF$\beta 1$ synthesis.

Reactive oxygen species (ROS) may constitute an additional mechanism whereby aldosterone produces renal vascular injury. Recently, Stier et al (49) studied SHRSP given $1 \% \mathrm{NaCl}$ drinking solution to which lisinopril ( $20 \mathrm{mg} / \mathrm{kg} /$ day) was added. All animals also received aldosterone at $40 \mu \mathrm{g} / \mathrm{kg} / \mathrm{day}$, subcutaneously, for 2 weeks. Four groups of animals were studied: 1) $1 \mathrm{ml} / \mathrm{kg} /$ day sterile saline, intraperitoneally (vehicle); 2) 250 $\mathrm{mg} / \mathrm{kg} /$ day BID dimethylthiourea (DMTU), a hydroxyl radical scavenger, intraperitoneally; 3) $250 \mathrm{mg} / \mathrm{kg} /$ day BID tempol, a superoxide dismutase mimetic, intraperitoneally; 4) DMTU and tempol together. Lisinopril-treated SHRSP infused with aldosterone developed marked proteinuria, thrombotic microangiopathy, and ischemic changes in tubules. Treatment with DMTU or DMTU and tempol, but not tempol alone, markedly reduced thrombotic microangiopathy and tubular damage compared to the animals receiving vehicle. These findings suggest an important role for ROS, in particular hydroxyl radicals, in mediating renal vascular injury and tubular damage produced by aldosterone.

Other potential mechanisms of pathogenetic nonepithelial effects of aldosterone include increased sodium influx in cultured VSMCs (44), and potentiation of the effects of catecholamines through inhibition of norepinephrine uptake in VSMCs (45) and myocardial cells (43).

Finally, Farquharson and Struthers (27) conducted elegant studies demonstrating that aldosterone produces endothelial dysfunction, perhaps by inhibiting nitric oxide release. Spironolactone-induced aldosterone blockade increased nitric oxide bioactivity, with a resultant improvement in endothelial vasodilator dysfunction (vide infra).

\section{Aldosterone receptor blockade: results from experimental models}

As detailed in a recent review (15), several animal models of aldosterone receptor blockade have demonstrated the ability of mineralocorticoid antagonism to retard the progression of vascular and myocardial fibrosis, even in the absence of lowered blood pressure. These include studies in several experimental models, including spontaneously hypertensive rats, the Goldblatt model of renovascular hypertension, and aldosterone models of acquired arterial hypertension (54-56).

\section{Aldosterone receptor blockade: results from clinical trials}

In recent years, clinical trials have begun to validate the ability of aldosterone receptor antagonism to favorably effect myocardial fibrosis in humans. Using serum measurements of procollagen type III amino terminal peptide (PIINP) - a serum marker of myocardial collagen turnover - MacFadyen et al (57) demonstrated that patients with congestive heart failure treated with a diuretic, an ACE inhibitor, and spironolactone had significantly reduced myocardial collagen turnover in comparison with patients treated with a diuretic, an ACE inhibitor, and placebo. This trial demonstrated the ability of endogenous aldosterone, which is present despite ACE inhibitor therapy, to stimulate myocardial fibrosis in human heart failure.

Farquharson and Struthers (27) demonstrated in an indirect manner that aldosterone could play a role in endothelial dysfunction in chronic heart failure. They performed a randomized, placebo-controlled, double-blind crossover study in 10 patients with New York Heart Association (NYHA) class II to III chronic heart failure on standard diuretic and ACE inhibitor therapy, comparing $50 \mathrm{mg} /$ day of spironolactone versus placebo for a period of 1 month. Forearm vascular endothelial function was assessed by bilateral forearm venous occlusion plethysmography using acetylcholine and N-monoethyl-L-arginine (L-NMMA), with sodium nitroprusside as a control vasodilator. The aldosterone antagonist spironolactone substantively increased the forearm blood flow response to acetylcholine compared with placebo, with an associated increase in vasoconstriction due to L-NMMA. Therefore, they concluded that antagonizing the aldosterone receptor improves endothelial dysfunction and increases nitric oxide bioactivity in chronic heart failure.

Recently, the Randomized Aldactone Evaluation Study (RALES) (16) examined the effect of spironolactone on overall morbidity and mortality in patients with severe heart failure treated with standard therapy with an ACE inhibitor, a loop diuretic, and digoxin combined with either a nonhemodynamic dose of spironolactone ( $25 \mathrm{mg} /$ day) or placebo. This seminal trial was discontinued early by the Data Safety Monitoring Board after a mean follow-up of 24 months, and disclosed that spironolactone-treated patients demonstrated a $30 \%$ reduction 
in the risk of death from all causes compared with the placebo group. This reduction in mortality was largely attributed to a reduction in death from progressive heart failure and sudden cardiac death. With respect to improvements in morbidity, patients in the spironolactone group had a $35 \%$ decreased frequency of hospitalization for worsening heart failure compared with patients in the placebo group and showed significant improvement in the symptoms of heart failure $(p<0.001)$. The RALES investigators attributed the beneficial actions of spironolactone to the drug's favorable effects on myocardial and vascular fibrosis and its ability to increase myocardial uptake of norepinephrine, in addition to its anticipated ability to prevent sodium retention and potassium loss.

\section{Animal Models Supporting a Renal-Protective Effect of Aldosterone Receptor Blockade}

While it may seem speculative to extrapolate the above experimental and clinical data of myocardial and vascular effects of aldosterone receptor antagonists to potential benefit in the kidney, this information does provide insight into the possible beneficial mechanisms of these agents in progressive renal disease. Recent experimental data have shown the ability of aldosterone receptor antagonists to attenuate proteinuria and renal damage. As described previously, Rocha et al (32) demonstrated that aldosterone receptor blockade with spironolactone markedly attenuated urinary protein excretion, as well as prevented the development of nephrosclerotic and cerebrovascular lesions, independent of changes in arterial blood pressure in saline-drinking SHRSP. Spironolactone, although an effective aldosterone receptor antagonist, also has affinity for other steroid receptors, thereby producing unwanted adverse effects.

Consequently, the same investigators recently conducted a second study using eplerenone, a selective aldosterone receptor antagonist (58). Using the same saline-drinking SHRSP model, investigators performed two experiments to evaluate whether eplerenone would provide the same renal-protective effects as spironolactone. In the first experiment, the impact of eplerenone on urinary protein excretion and blood pressure was compared with a control group following infusions of aldosterone or angiotensin II. Eplerenone prevented proteinuria (15 $\mathrm{mg} /$ day for eplerenone vs $92 \mathrm{mg} /$ day for the control group, $\mathrm{p}<0.001$ ) and renal lesions ( $2 \%$ for eplerenone vs $40 \%$ for the control group, $\mathrm{p}<0.0005)$. However, there were no statistically significant differences between the eplerenone and control groups in blood pressure $(226 \mathrm{mmHg}$ vs $234 \mathrm{mmHg}$, respectively). In the second experiment, five groups were studied: vehicle (control), captopril, captopril followed by an aldosterone infusion, captopril followed by an angiotensin II infusion, and the combination of captopril and eplerenone followed by an angiotensin II infusion. After 2 weeks, proteinuria $(158 \mathrm{mg} /$ day, $121 \mathrm{mg} /$ day, $96 \mathrm{mg} /$ day vs $16 \mathrm{mg} /$ day, respectively; $\mathrm{p}<0.001)$ and glomerular $(18 \%, 15 \%, 16 \%$ vs $0 \%$, respectively; $\mathrm{p}<0.001)$ and renal vascular lesions $(24 \%, 26 \%, 17 \%$ vs $0 \%$, respectively; $\mathrm{p}<0.001)$ were significantly higher in the control, captopril plus aldosterone, and captopril plus angiotensin
II groups versus the captopril alone group. Thus, both the aldosterone and angiotensin II infusions reversed the renal-protective effects of captopril. However, when the captopril plus angiotensin II group was compared with the combination of captopril, eplerenone, and angiotensin II, the latter combination group had substantially less proteinuria $(96 \mathrm{mg} /$ day vs 28 $\mathrm{mg} /$ day, respectively, $\mathrm{p}<0.001$ ), and significantly fewer glomerular $(16 \%$ vs $4 \%$, respectively, $\mathrm{p}<0.001)$ and renal vascular lesions $(17 \%$ vs $4 \%$, respectively, $\mathrm{p}<0.001)$. In contrast to the reversal of renal protection seen when angiotensin II was added to captopril treatment, the addition of eplerenone to the aforementioned regimen attenuated proteinuria and renal damage in SHRSP. After 2 weeks of treatment, mean systolic blood pressures were elevated but not statistically different among the five treatment groups. In analogy with previous findings with spironolactone, eplerenone conferred renal protection independently of its effect on blood pressure.

\section{Therapeutic Implications}

Initially, one might anticipate that the adverse effects of aldosterone could be mitigated merely by blocking aldosterone synthesis utilizing ACE inhibitors or angiotensin II receptor blockers. This unfortunately is not the case. Several investigators have demonstrated that the use of ACE inhibitors initially causes an acute decrease in the concentration of aldosterone, but with continued use, this suppression is not sustained (5962).

In a recent study, Sato et al (63) systematically examined the relationship between plasma aldosterone concentrations and the degree of ACE inhibition in 25 essential hypertensive patients treated with an ACE inhibitor. Blood pressure decreased with treatment and plasma ACE activity was suppressed compared with that of hypertensive patients treated with medication other than ACE inhibitors. No relationship was found between the level of ACE inhibition and plasma aldosterone concentrations, which rose in parallel with the duration of ACE inhibitor treatment. Thus, their study demonstrated that although continuous ACE inhibitor therapy produces significant suppression of plasma ACE activity in essential hypertensive patients, no relationship exists between levels of ACE inhibition and concomitant plasma aldosterone concentrations. They clearly demonstrated that plasma aldosterone concentrations tend to increase with the duration of ACE inhibitor treatment, despite a persistent and sustained inhibition of ACE activity.

In conclusion, the available evidence clearly indicates that therapy with ACE inhibitors to suppress aldosterone (in addition to the harmful effects of angiotensin II) is not adequate. It has been proposed that the use of aldosterone receptor antagonists, in addition to ACE inhibitors, will have additional benefit in the prevention of end-organ damage $(59,60)$.

\section{Hyperkalemia as a potential limiting factor to aldosterone re- ceptor antagonist therapy}

An important consideration regarding the feasibility of aldosterone receptor antagonist therapy is the risk of provoking hyperkalemia. Many patients with chronic renal disease are 
already receiving an ACE inhibitor or an angiotensin II (type 1) receptor antagonist, which would enhance the risk of hyperkalemia.

Despite such concerns, the experience with the RALES trial is somewhat reassuring. In that study, patients already on ACE inhibitor therapy who were then randomized to spironolactone experienced a $0.3 \mathrm{mmol} / \mathrm{l}$ increase in median potassium concentrations. Admittedly, the doses of spironolactone used were modest. Although the difference between the spironolactone and placebo groups achieved statistical significance $(\mathrm{p}<0.001)$, the increase was not considered clinically important (16). Only further investigation including rigorous clinical trials will definitively address these concerns.

\section{Concomitant treatment with ACE inhibitors and mineralocor- ticoid antagonists}

A recent study by Sato et al (64) exemplifies the potential benefits of a therapeutic regimen encompassing the concomitant administration of ACE inhibitors and aldosterone antagonists. These investigators analyzed the association between cardiac hypertrophy and aldosterone in 29 patients (18 patients with nondiabetic nephropathy and 11 patients with diabetic nephropathy) who developed end-stage renal disease treated by hemodialysis. Among the nondiabetic patients, the left ventricular mass index correlated significantly with plasma aldosterone concentrations both before and after hemodialysis, but it did not correlate with plasma renin activity. Furthermore, left ventricular mass index also correlated with mean blood pressure. In contrast, these correlations were not demonstrated in the diabetic patients, despite similar age distribution, duration of hemodialysis, and several echocardiographic parameters between the two groups. They interpreted their data as indicating evidence for a relation between left ventricular hypertrophy and plasma aldosterone concentrations that is independent of blood pressure in nondiabetic patients with endstage renal failure treated with hemodialysis.

\section{Limitations of nonselective aldosterone receptor blockade}

While an effective antialdosterone agent, widespread use of spironolactone in humans is limited by its tendency to produce undesirable sexual side effects. At standard doses, impotence and gynecomastia can be induced in men, while premenopausal women may experience menstrual disturbances. These adverse effects are due to the binding of spironolactone to progesterone and androgen receptors, and are a substantial cause of drug discontinuation. In a study involving 43 patients treated with long-term spironolactone for mineralocorticoid excess syndromes, 13 patients $(30 \%)$ were switched to alternate therapy due to the occurrence of gynecomastia (6/20 males), and menstrual disturbances or breast pain (7/23 females) (65). The RALES trial (16) reported a 10\% incidence of gynecomastia or breast pain in its male subjects (patients in this trial received 25 to $50 \mathrm{mg}$ /day of spironolactone). This incidence was significantly higher than placebo $(10 \%$ vs $1 \%, \mathrm{p}<0.001)$ and caused significantly more patients to discontinue treatment ( $2 \%$ vs $0.2 \%, \mathrm{p}=0.006$ ). While troublesome, these side effects have been shown to be reversible and doserelated. At doses $\leq 50 \mathrm{mg}$ / day, the incidence of gynecomastia is $6.9 \%$, but rises to $52 \%$ as doses are increased to greater than $150 \mathrm{mg} /$ day (66). Moreover, this adverse effect exhibits a faster onset at higher doses (67). Other studies in women taking spironolactone for dermatologic disorders reiterate the dose relationship of these sexual side effects; doses substantially greater than $100 \mathrm{mg} /$ day are more frequently associated with menstrual disturbances and breast enlargement $(68,69)$. The advent of selective aldosterone receptor antagonists should demonstrate a reduction in these side effects, and thus, lead to an improvement in patient compliance with antialdosterone therapy.

\section{Selective aldosterone receptor antagonists (SARAs)}

Preliminary data on the selective aldosterone receptor antagonist (SARA), eplerenone, appear promising for the effective blockade of aldosterone - and its harmful effects- without the sexual disturbances of spironolactone. This agent is currently in Phase III trials for the treatment of hypertension and heart failure. Eplerenone has demonstrated a much lower affinity for androgen and progesterone receptors than spironolactone, but has twice the potency with respect to antimineralocorticoid activity (70). As mentioned previously, animal models have demonstrated the ability of eplerenone to prevent the occurrence of proteinuria and renal lesions in saline-drinking SHRSP (58). Furthermore, in the rat model of ascending aortic stenosis, investigators examined the effect of eplerenone versus no drug on left ventricular (LV) hypertrophy in the presence of severe pressure overload. Despite similar elevations in LV pressure, rats treated with eplerenone had significantly lower heart weight, LV end diastolic pressure, and LV mass compared with untreated rats ( $<<0.05$ for all comparisons) (71).

Early clinical studies $(72,73)$ support the concept that eplerenone has similar efficacy to spironolactone, without exhibiting antiandrogenic adverse effects. In patients with mild to moderate hypertension, eplerenone provided comparable blood pressure reductions to spironolactone, which were sustained over the 24-hour dosing period. The incidence of adverse effects with eplerenone was similar to that of placebo, with no reports of gynecomastia (72). In patients with NYHA class II to IV heart failure, both eplerenone and spironolactone produced significant decreases in brain natriuretic peptide, and increased urinary aldosterone and plasma renin compared with placebo. Consistent with the lower affinity of eplerenone for androgen and progesterone receptors seen in animal studies, male patients receiving spironolactone experienced significant increases in testosterone $(\mathrm{p} \leq 0.02)$ compared with eplerenone (73).

\section{Summary and Potential Clinical Implications}

Recent observations indicate that it is no longer appropriate to consider that the endocrine or paracrine properties of aldosterone are restricted to what has been called "classic target cells". The hemodynamic and humoral actions of aldosterone have important clinical implications for the pathogenesis of 
progressive renal disease, and consequently, may influence future antihypertensive strategies. Although ACE inhibitors are very effective in retarding disease progression, theoretically, there may be additional benefit achieved with concurrent aldosterone receptor blockade. As observed in recent clinical studies of congestive heart failure, as well as in animal models of renal disease, antagonism of aldosterone protects against endorgan damage through both hemodynamic and direct cellular actions. With the advent of selective aldosterone receptor antagonists, it is now feasible to conduct "proof of concept" antihypertensive studies to assess whether end-organ damage, including progressive renal disease, can be more effectively prevented, without the dose-limiting side effects of nonspecific aldosterone receptor blockade. The demonstration that selective aldosterone receptor blockade would retard progression of renal disease would constitute an important platform for advocating the addition of aldosterone antagonists to the therapeutic regimen used for attenuation of progressive renal dysfunction.

\section{References}

1) U.S. Renal Data System. Précis. A summary of the United States ESRD program. Incidence and Prevalence, Chapter 1. USRDS 2000 Annual Data Report, National Institutes of Health, National Institute of Diabetes and Digestive and Kidney Diseases, Bethesda, MD, June 2000: 15-54.

2) U.S. Renal Data System. USRDS 1997 Annual Report, National Institutes of Health, National Institute of Diabetes and Digestive and Kidney Disease, Bethesda, MD, 1997: 21-34.

3) U.S. Renal Data System. International Comparisons, Chapter 12. USRDS 2000 Annual Data Report, National Institutes of Health, National Institute of Diabetes and Digestive and Kidney Diseases, Bethesda, MD, June 2000: 177-182.

4) Egido J. Vasoactive hormones and renal sclerosis. Kidney Int 49: 578597, 1996.

5) Ibrahim HN, Rosenberg ME, Hostetter TH. Role of the renin angiotensinaldosterone system in the progression of renal disease: a critical review. Semin Nephrol 17: 431-440, 1997.

6) Remuzzi G, Ruggenenti P, Benigni A. Understanding the nature of renal disease progression. Kidney Int 51: 2-15, 1997.

7) The GISEN Group (Gruppo Italiano di Studi Epidemiologici in Nefrologia). Randomised placebo-controlled trial of effect of ramipril on decline in glomerular filtration rate and risk of terminal renal failure in proteinuric, non-diabetic nephropathy. Lancet 349: 1857-1863, 1997.

8) Lewis EJ, Hunsicker LG, Bain RP, Rohde RD, and The Collaborative Study Group. The effect of angiotensin-converting-enzyme inhibition on diabetic nephropathy. N Engl J Med 329: 1456-1462, 1993 (published erratum appears in N Engl J Med 330: 152, 1993).

9) Gavras H, Brunner HR, Laragh JH, et al Malignant hypertension resulting from deoxycorticosterone acetate and salt excess: role of renin and sodium in vascular changes. Circ Res 36: 300-309, 1975.

10) Greene EL, Kren S, Hostetter TH. Role of aldosterone in the remnant kidney model in the rat. J Clin Invest 98: 1063-1068, 1996.

11) Horiuchi $M$, Nishiyama $H$, Hama $J$, et al Characterization of renal aldosterone receptors in genetically hypertensive rats. Am J Physiol 264: F286F291, 1993.

12) Rocha R, Chander PN, Zuckerman A, Stier CT Jr. Role of aldosterone in renal vascular injury in stroke-prone hypertensive rats. Hypertension 33: 232-237, 1999.

13) Quan ZY, Walser M, Hill GS. Adrenalectomy ameliorates ablative nephropathy in the rat independently of corticosterone maintenance level. Kidney Int 41: 326-333, 1992.

14) Ullian ME, Islam MM, Robinson CJ, Fitzgibbon WR, Tobin ET, Paul RV.
Resistance to mineralocorticoids in Wistar-Furth rats. Am J Physiol 272: H1454-H1461, 1997.

15) Epstein M. Aldosterone as a mediator of progressive renal disease: pathogenetic and clinical implications. Am J Kidney Dis 37: 677-688, 2001.

16) Pitt B, Zannad F, Remme WJ, et al Randomized Aldactone Evaluation Study Investigators. The effect of spironolactone on morbidity and mortality in patients with severe heart failure. N Engl J Med 341: 709-717, 1999.

17) Anderson S, Rennke HG, Brenner BM. Therapeutic advantage of converting enzyme inhibitors in arresting progressive renal disease associated with systemic hypertension in the rat. J Clin Invest 77: 1993-2000, 1986.

18) Ritz E, Orth S, Weinreich T, Wagner J. Systemic hypertension versus intraglomerular hypertension in progression. Kidney Int 45: 438-442, 1994.

19) Meyer TW, Anderson S, Rennke HG, Brenner BM. Reversing glomerular hypertension stabilizes established glomerular injury. Kidney Int 31: 752-759, 1987.

20) Ruggenenti P, Perna A, Gherardi G, Gaspari F, Benini R, Remuzzi G, and Gruppo Italiano di Studi Epidemiologici in Nefrologia (GISEN). Renal function and requirement for dialysis in chronic nephropathy patients on long-term ramipril: REIN follow-up trial. Ramipril Efficacy in Nephropathy. Lancet 352: 1252-1256, 1998.

21) Maschio G, Alberti D, Janin G, et al Effect of the angiotensin-convertingenzyme inhibitor benazepril on the progression of chronic renal insufficiency. The Angiotensin-Converting-Enzyme Inhibition in Progressive Renal Insufficiency Study Group. N Engl J Med 334: 939-945, 1996.

22) Berl T, Katz FH, Henrich WL, de Torrente A, Schrier RW. Role of aldosterone in the control of sodium excretion in patients with advanced chronic renal failure. Kidney Int 14: 228-235, 1978.

23) Hene RJ, Boer P, Koomans HA, Mees EJ. Plasma aldosterone concentrations in chronic renal disease. Kidney Int 21: 98-101, 1982.

24) Foster $\mathrm{RH}$, MacFarlane $\mathrm{CH}$, Bustamante MO. Recent progress in understanding aldosterone secretion. Gen Pharmacol 28: 647-651, 1997.

25) Fuller PJ. Aldosterone's effects and mechanism of action. Current Opinion in Endocrinology of Diabetes 4: 218-224, 1997.

26) Luetscher JA Jr, Neher JP, Wettstein A. Isolation of crystalline aldosterone from the urine of a nephrotic patient. Experientia 10: 456-458, 1954.

27) Farquharson CA, Struthers AD. Spironolactone increases nitric oxide bioactivity, improves endothelial vasodilator dysfunction, and suppresses vascular angiotensin I/angiotensin II conversion in patients with chronic heart failure. Circulation 101: 594-597, 2000.

28) Duprez D, De Buyzere M, Rietzchel ER, Clement DL. Aldosterone and vascular damage. Current Hypertension Reports 2: 327-334, 2000.

29) Conn JW, Knopf RF, Nesbit RM. Clinical characteristics of primary aldosteronism from an analysis of 145 cases. Am J Surg 107: 159-172, 1964.

30) Nishimura M, Uzu T, Fujii T, et al Cardiovascular complications in patients with primary aldosteronism. Am J Kidney Dis 33: 261-266, 1999.

31) Young SC, Shionoiri H, Takasaki I, Kihara M, Gotoh E. Hypertensive complications in patients with primary aldosteronism. A retrospective study. Curr Ther Res 50: 317-325, 1991.

32) Rocha R, Chander PN, Khanna K, Zuckerman A, Stier CT Jr. Mineralocorticoid blockade reduces vascular injury in stroke-prone hypertensive rats. Hypertension 31: 451-458, 1998.

33) Hostetter TH, Kren S, Ibrahim HN. Mineralocorticoid receptor blockade in the remnant kidney model. J Am Soc Nephrol 10: 85A, 1999 (Abstract).

34) Brilla CG, Weber KT. Mineralocorticoid excess, dietary sodium and myocardial fibrosis. J Lab Clin Med 120: 893-901, 1992.

35) Ullian ME, Schelling JR, Linas SL. Aldosterone enhances angiotensin II receptor binding and inositol phosphate responses. Hypertension 20: 6773, 1992.

36) Young M, Fullerton M, Dilley R, Funder J. Mineralocorticoids, hypertension, and cardiac fibrosis. J Clin Invest 93: 2578-2583, 1994.

37) Bonvalet JP, Alfaidy N, Farman N, Lombes M. Aldosterone: intracellular receptors in human heart. Eur Heart J 16 suppl N: 92-97, 1995. 


\section{Aldosterone and Progressive Nephropathy}

38) Kornel L. Colocalization of 11 beta-hydroxysteroid dehydrogenase and mineralocorticoid receptors in cultured vascular smooth muscle cells. Am J Hypertens 7: 100-103, 1994.

39) Lombes M, Oblin ME, Gasc JM, Baulieu EE, Farman N, Bonvalet JP. Immunohistochemical and biochemical evidence for a cardiovascular mineralocorticoid receptor. Circ Res 71: 503-510, 1992.

40) Tanaka J, Fujita H, Matsuda S, Toku K, Sakanaka M, Maeda N. Glucocorticoid- and mineralocorticoid receptors in microglial cells: the two receptors mediate differential effects of corticosteroids. Glia 20: 23-37, 1997.

41) Hatakeyama H, Miyamori I, Fujita T, Takeda Y, Takeda R, Yamamoto H. Vascular aldosterone. Biosynthesis and a link to angiotensin II-induced hypertrophy of vascular smooth muscle cells. J Biol Chem 269: 2431624320, 1994.

42) Silvestre JS, Robert V, Heymes C, et al Myocardial production of aldosterone and corticosterone in the rat. Physiological regulation. J Biol Chem 273: 4883-4891, 1998.

43) Barr CS, Lang CC, Hanson J, Arnott M, Kennedy N, Struthers AD. Effects of adding spironolactone to an angiotensin-converting enzyme inhibitor in chronic congestive heart failure secondary to coronary artery disease. Am J Cardiol 76: 1259-1265, 1995.

44) Kornel L, Smoszna-Konaszewska B. Aldosterone (ALDO) increases transmembrane influx of $\mathrm{Na}^{+}$in vascular smooth muscle (VSM) cells through increased synthesis of $\mathrm{Na}^{+}$channels. Steroids 60: 114-119, 1995.

45) Weber MA, Purdy RE. Catecholamine-mediated constrictor effects of aldosterone on vascular smooth muscle. Life Sci 30: 2009-2017, 1982.

46) Stier CT, Chander PN, Zuckerman A, Rocha R. Nonepithelial effects of aldosterone. Current Opinion in Endocrinology of Diabetes 5: 211-216, 1998.

47) Brown NJ, Kim KS, Chen YQ, et al Synergistic effect of adrenal steroids and angiotensin II on plasminogen activator inhibitor-1 production. J Clin Endocrinol Metab 85: 336-344, 2000.

48) Brown NJ, Nakamura S, Ma L, et al Aldosterone modulates plasminogen activator inhibitor- 1 and glomerulosclerosis in vivo. Kidney Int 58: 1219 $1227,2000$.

49) Stier C Jr, Zuckerman A, Harashima $\mathrm{H}$, Chander P. Antioxidants reduce aldosterone-induced renal vascular injury in stroke-prone spontaneously hypertensive rats (Abstract P4.03). Proceedings of the International Society of Hypertension. August 23, 2000, Chicago, IL.

50) Sun Y, Zhang J, Zhang JQ, Ramires FJ. Local angiotensin II and transforming growth factor-beta1 in renal fibrosis of rats. Hypertension 35: 1078-1084, 2000.

51) Weber KT, Anversa P, Armstrong PW, et al Remodeling and reparation of the cardiovascular system. J Am Coll Cardiol 20: 3-16, 1992.

52) Nakamura $S$, Nakamura $I$, Ma $L$, Vaughan $D E$, Fogo AB. Plasminogen activator inhibitor- 1 expression is regulated by the angiotensin type 1 receptor in vivo. Kidney Int 58: 251-259, 2000.

53) Brown NJ, Agirbasli MA, Williams GH, Litchfield WR, Vaughan DE. Effect of activation and inhibition of the renin-angiotensin system on plasma PAI-1. Hypertension 32: 965-971, 1998.

54) Nicoletti A, Heudes $D$, Hinglais $N$, et al Left ventricular fibrosis in renovascular hypertensive rats. Effect of losartan and spironolactone. Hypertension 26: 101-111, 1995.

55) Benetos A, Lacolley P, Safar ME. Prevention of aortic fibrosis by spironolactone in spontaneously hypertensive rats. Arterioscler Thromb Vasc Biol
17: 1152-1156, 1997.

56) Brilla CG, Matsubara LS, Weber KT. Antifibrotic effects of spironolactone in preventing myocardial fibrosis in systemic arterial hypertension. Am J Cardiol 71: 12A-16A, 1993.

57) MacFadyen RJ, Barr CS, Struthers AD. Aldosterone blockade reduces vascular collagen turnover, improves heart rate variability and reduces early morning rise in heart rate in heart failure patients. Cardiovasc Res 35: 30-34, 1997.

58) Rocha R, Chander PN, Zuckerman A, Stier CT. Role of mineralocorticoids in renal injury in stroke-prone hypertensive rates. Hypertension 32: 598, 1998 (Abstract).

59) Pitt B. "Escape" of aldosterone production in patients with left ventricular dysfunction treated with an angiotensin converting enzyme inhibitor: implications for therapy. Cardiovasc Drugs Ther 9: 145-149, 1995.

60) Struthers AD. Aldosterone escape during angiotensin-converting enzyme inhibitor therapy in chronic heart failure. J Card Fail 2: 47-54, 1996.

61) Borghi C, Boschi S, Ambrosioni E, Melandri G, Branzi A, Magnani B. Evidence of a partial escape of renin-angiotensin-aldosterone blockade in patients with acute myocardial infarction treated with ACE inhibitors. J Clin Pharmacol 33: 40-45, 1993.

62) Staessen J, Lijnen P, Fagard R, Verschueren LJ, Amery A. Rise in plasma concentration of aldosterone during long-term angiotensin II suppression. J Endocrinol 91: 457-465, 1981.

63) Sato A, Suzuki Y, Shibata H, Saruta T. Plasma aldosterone concentrations are not related to the degree of angiotensin-converting enzyme inhibition in essential hypertensive patients. Hypertens Res 23: 25-31, 2000.

64) Sato A, Funder JW, Saruta T. Involvement of aldosterone in left ventricular hypertrophy of patients with end-stage renal failure treated with hemodialysis. Am J Hypertens 12: 867-873, 1999.

65) Mantero F, Opocher G, Rocco S, Carpene G, Armanini D. Long-term treatment of mineralocorticoid excess syndromes. Steroids 60: $81-86$, 1995.

66) Jeunemaitre $X$, Chatellier G, Kreft-Jais $C$, et al Efficacy and tolerance of spironolactone in essential hypertension. Am J Cardiol 60: 820-825, 1987.

67) de Gasparo M, Whitebread SE, Preiswerk G, Jeunemaitre X, Corvol P, Menard J. Antialdosterones: incidence and prevention of sexual side effects. J Steroid Biochem 32: 223-227, 1989.

68) Helfer EL, Miller JL, Rose LI. Side-effects of spironolactone therapy in the hirsute woman. J Clin Endocrinol Metab 66: 208-211, 1988.

69) Hughes BR, Cunliffe WJ. Tolerance of spironolactone. Br J Dermatol 118: 687-691, 1988.

70) Rabasseda X, Silvestre J, Castañer J. Eplerenone. Drugs Future 24: 488501, 1999.

71) Hasan F, Weinberg EO, Delyani J, et al. Aldosterone and cardiac hypertrophy: effects of eplerenone on LV function and hypertrophic remodeling in LV pressure overload. Circulation 100 (suppl I): I-565, 1999 (Abstract).

72) Epstein M, Alexander JC, Roniker B. Efficacy and safety of eplerenone, a novel and selective aldosterone receptor antagonist (SARA), in patients with mild to moderate hypertension. Hypertension 33: 1075, 1999 (Abstract).

73) Pitt B, Roniker B. Eplerenone, a novel selective aldosterone receptor antagonist (SARA): a dose finding study in patients with heart failure. J Am Coll Cardiol 33 (suppl A): 188A-189A, 1999 (Abstract). 